\title{
Electronic Structure of Some Wurtzite Semiconductors: Hybrid Functionals vs. Ab Initio Many Body Calculations
}

\author{
J. KACZKOWSKI* \\ Institute of Molecular Physics, Polish Academy of Sciences \\ M. Smoluchowskiego 17, 60-179 Poznań, Poland
}

\begin{abstract}
Using the first-principles projector augmented wave method, the structural and electronic properties of wurtzite crystals, AlN, GaN, InN and $\mathrm{ZnO}$ have been calculated. Different exchange-correlation approximations: $\mathrm{LDA}, \mathrm{LDA}+U, \mathrm{GGA}, \mathrm{GGA}+U$ and hybrid Heyd-Scuseria-Ernzerhof method were used. We also present the values of band gap calculated within different $\mathrm{GW}$ approximations $\left(\mathrm{G}_{0} \mathrm{~W}_{0}, \mathrm{GW}_{0}\right.$, $\mathrm{GW}$ and $U+\mathrm{G}_{0} \mathrm{~W}_{0}$, the last one for materials with shallow $d$ states). In case of structural parameters the best agreement with experiment was obtained for hybrid Heyd-Scuseria-Ernzerhof functional and in case of band gap the best agreement was for GW and $U+\mathrm{G}_{0} \mathrm{~W}_{0}$ approximation.
\end{abstract}

PACS: 71.15.Mb, 71.20.-b, 71.20.Nr

\section{Introduction}

Density functional theory (DFT) [1] is currently the most popular method for calculating electronic and structural properties of solids. The computational scheme is based on the Kohn-Sham approach [2] in which the interacting system of electrons is mapped onto non-interacting one in such a way that electron density of both systems are the same. As result we obtain the Kohn-Sham equations in which the only unknown quantity is so-called exchange-correlation potential. The most popular approximations (local density LDA [3] and generalized gradient GGA [4]) for this term have been derived from homogeneous electron gas model.

Results obtained within both approximations in many cases are in reasonable agreement with experimental data. But there are different types of materials in which this approach for exchange-correlation potential is insufficient, for example: transition-metal oxides (e.g. $\mathrm{NiO}$ ) in which wrong magnetic and electronic state can be predicted and semiconductors in which the main problem arises when we try to calculate band-gap. To avoid this problem some other approximations have been proposed, like for example LDA $+U$ based on the Hubbard model for strongly correlated electrons [5]. In this method the on-site $d-d$ Coulomb interaction $U$ is added to the LDA (or GGA) functional. The GW approximation [6] based on many-body perturbation theory is widely used method to predict band-gap of semiconductors and insulators. In this approach the self-energy in terms of single particle

\footnotetext{
* e-mail: kaczkowski@ifmpan.poznan.pl
}

Green's function G and screened Coulomb interaction is calculated on top of LDA results. Single calculation of this parameters is called the $\mathrm{G}_{0} \mathrm{~W}_{0}$ approximation and it yields improved value of the band gap, but in many cases (e.g. zinc oxide $\mathrm{ZnO}$ ) this value is still too small [6].

Better results are obtained by self-consistent GW calculations. In partially self-consistent $\mathrm{GW}_{0}$ only $\mathrm{G}$ is iterating and $\mathrm{W}$ is fixed to the initial $\mathrm{W}_{0}$ taken from DFT. In fully self-consistent $\mathrm{GW}$ both $\mathrm{G}$ and $\mathrm{W}$ are updated. There is also possibility to combine LDA $+U$ approach with GW, and made the latter on top of the former $\left(U+\mathrm{G}_{0} \mathrm{~W}_{0}\right)$ which, according to our knowledge have not been applied for wurtzite nitrides yet. The last class of approximations are so-called hybrid functional [7] in which some part LDA/GGA exchange functional is replaced by exact exchange calculated within the HartreeFock approximation.

In case of solids the most popular one is based on formula suggested in [8] and it is called HSE (HeydScuseria-Ernzerhof). According to our knowledge there is no hybrid HSE calculations of band structures for wurtzite AlN and GaN. All mentioned approximations have been used to calculate band-gap of some wurtzite semiconductors (III-N and $\mathrm{ZnO}$ ) and the LDA/ $\operatorname{GGA}(+U)$ and HSE have been also used to obtain the lattice constant. This paper is organized as follows: in next section the short description of the method of calculation is presented, further we present results and discussion and finally short summation is given.

\section{Method of calculations}

All calculations were done by using the projector augmented wave method as implemented in Vienna ab ini- 
tio Simulation Package (VASP) [9, 10]. Both local density [3] and generalized gradient [4] approximations were used. The latter one was used for hybrid functional and GW calculations. The Brillouin zone integrations were performed by using $6 \times 6 \times 6 \Gamma$-centered $k$-point grid in all cases. The self-consistent GW calculations were performed up to eight iterations but self-consistency was obtained after fourth-fifth iteration. The total number of bands was always 150 in all cases for simplicity (as in [6]). In case of $\mathrm{DFT}+U$ calculations the values of $U$ parameter were taken from [11]: $U_{\mathrm{Zn}}=4.7 \mathrm{eV}, U_{\mathrm{Ga}}=3.9 \mathrm{eV}$ and $U_{\text {In }}=1.9 \mathrm{eV}$. The theoretical justification of this values for fully occupied cation $d$ states was given also in [11].

\section{Results}

In Table I the optimized lattice parameters were given. As could be expected, the LDA underestimates the lattice constants up to $1.5 \%$ in case of nitrides and $1.8 \%$ in case of $\mathrm{ZnO}$ while the GGA overestimates it by $1 \%$. The $\mathrm{LDA}+U$ gives larger error than $\mathrm{LDA}(3.5 \%$ in case of $\mathrm{ZnO}$ ) but $\mathrm{GGA}+U$ gives better agreement with experimental values (error $0.5 \%$ for $\mathrm{ZnO}$ and $\mathrm{InN}$ ). Both $\mathrm{LDA}+U$ and $\mathrm{GGA}+U$ give smaller values than LDA/GGA. This is connected with the fact that in LDA(GGA) $+U d$ electrons are more localized [11]. The best agreement with experimental results were obtained by using hybrid functional HSE. The error was smaller than $0.3 \%$. In case of $c / a$ ratios the error is less than $1 \%$.

TABLE I

Calculated lattice constants $a$ and $c / a$ ratios for $\mathrm{ZnO}$ and III-N within different exchange-correlation functionals. Experimental data were taken from [11]. In parentheses other available results for comparison are given: LDA and LDA $+U$ from [11], for III-N GGA and HSE from [12] and $\mathrm{ZnO}$ [13].

\begin{tabular}{c|c|c|c|c|c|c}
\hline \hline \multicolumn{7}{c}{$a[\AA]$} \\
\hline & LDA & LDA $+U$ & GGA & GGA $+U$ & HSE & Exp. \\
\hline \multirow{2}{*}{$\mathrm{ZnO}$} & 3.190 & 3.136 & 3.287 & 3.231 & 3.249 & 3.249 \\
& $(3.195)$ & $(3.148)$ & $(3.286)$ & & $(3.261)$ & \\
\hline \multirow{2}{*}{$\mathrm{AlN}$} & 3.090 & - & 3.130 & - & 3.103 & 3.110 \\
& $(3.090)$ & & $(3.130)$ & & $(3.103)$ & \\
\hline \multirow{2}{*}{$\mathrm{GaN}$} & 3.153 & 3.094 & 3.213 & 3.154 & 3.180 & 3.190 \\
& $(3.152)$ & $(3.094)$ & $(3.210)$ & & $(3.177)$ & \\
\hline \multirow{2}{*}{$\mathrm{InN}$} & 3.502 & 3.484 & 3.581 & 3.563 & 3.542 & 3.533 \\
& $(3.507)$ & $(3.488)$ & $(3.590)$ & & $(3.548)$ & \\
\hline \multicolumn{7}{|c|}{$c / a$} \\
$\mathrm{ZnO}$ & 1.620 & 1.614 & 1.613 & 1.615 & 1.614 & 1.603 \\
& $(1.615)$ & $(1.612)$ & $(1.613)$ & & $(1.602)$ & \\
\hline \multirow{2}{*}{$\mathrm{AlN}$} & 1.601 & - & 1.601 & - & 1.601 & 1.601 \\
& $(1.602)$ & \multicolumn{1}{c}{$(1.610)$} & & $(1.607)$ & \\
\hline \multirow{2}{*}{$\mathrm{GaN}$} & 1.631 & 1.629 & 1.631 & 1.628 & 1.624 & 1.627 \\
& $(1.631)$ & $(1.629)$ & $(1.630)$ & & $(1.626)$ & \\
\hline \multirow{2}{*}{$\mathrm{InN}$} & 1.616 & 1.614 & 1.616 & 1.615 & 1.609 & 1.611 \\
& $(1.618)$ & $(1.617)$ & $(1.620)$ & & $(1.621)$ & \\
& & & & & \\
\hline
\end{tabular}

In Table II the calculated band gap values were given. Again as could be expected, these values are seriously underestimated. The largest error in case of $\mathrm{ZnO}, \mathrm{GaN}$ and $\mathrm{InN}$ is caused by overestimation of localization of cation $d$ states which yields to strong $p-d$ hybridization and as result the smaller band gap (or metallic state for $\mathrm{InN}$ ). After using non-self-consistent $\mathrm{G}_{0} \mathrm{~W}_{0}$ approach the band gaps are still underestimated. Using GGA $+U$ improves the values of band gap but error is still large. Applying $U+\mathrm{G}_{0} \mathrm{~W}_{0}$ gives values close to experimental ones. Due to the fact that GW method is implemented in VASP only for semiconductors (at the DFT level) this method could not be applied for InN which is metallic from GGA point of view. HSE gives good estimation of band gap in case of $\mathrm{GaN}$ and $\mathrm{InN}$ but for $\mathrm{ZnO}$ and $\mathrm{AlN}$ the error is similar to $\mathrm{G}_{0} \mathrm{~W}_{0}$ calculation.

TABLE II

Calculated direct band gaps for $\mathrm{ZnO}$ and III-N by using different approximations for exchange-correlation functionals. Experimental data were taken from [11]. In parentheses other available results for comparison: LDA and $\mathrm{LDA}+U$ [11], hybrid HSE for III-N [12] and $\mathrm{ZnO}$ [13], $\mathrm{G}_{0} \mathrm{~W}_{0}$ for III-N [14] and $\mathrm{ZnO}[15]$.

\begin{tabular}{c|c|c|c|c|c|c}
\hline \hline \multicolumn{7}{c}{ Band gap [eV] } \\
\hline & GGA & GGA $+U$ & HSE & $\mathrm{G}_{0} \mathrm{~W}_{0}$ & $U+\mathrm{G}_{0} \mathrm{~W}_{0}$ & Exp. \\
\hline \multirow{2}{*}{$\mathrm{ZnO}$} & 0.793 & 1.403 & 2.499 & 2.334 & 3.152 & 3.430 \\
& $(0.800)$ & $(1.510)$ & $(2.480)$ & $(2.440)$ & & \\
\hline \multirow{2}{*}{$\mathrm{AlN}$} & 4.095 & - & 5.714 & 5.523 & - & 6.190 \\
& $(4.410)$ & & $(5.610)$ & $(5.800)$ & & \\
\hline \multirow{2}{*}{$\mathrm{GaN}$} & 1.774 & 2.489 & 3.348 & 2.911 & 3.777 & 3.500 \\
& $(2.140)$ & $(2.870)$ & $(3.230)$ & $(3.500)$ & & \\
\hline \multirow{2}{*}{$\mathrm{InN}$} & -0.160 & 0.000 & 0.772 & - & - & $0.7-0.8$ \\
& $(-0.180)$ & $(0.030)$ & $(0.630)$ & & &
\end{tabular}

In Table III the values of band gap within GGA and different GW approximations were given. Single $\mathrm{G}_{0} \mathrm{~W}_{0}$ on top of GGA calculations improves these values but they are still too small compared to experimental one. This understimation is about $1,0.5$, and $0.15 \mathrm{eV}$ for $\mathrm{ZnO}$, $\mathrm{AlN}$, and GaN, respectively. The large underestimation in case $\mathrm{ZnO}$ could be connected with incomplete cancellation of the Coulomb self-interaction within localized $d$ states [6]. For GaN this error is smaller due to the fact that $d$ electrons lie much deeper than in $\mathrm{ZnO}$. The different behaviour is observed for $\mathrm{GW}_{0}$ approximation: for $\mathrm{ZnO}$ band gap value is slightly improved (about $0.5 \mathrm{eV}$ ), for AlN this value almost does not change, and for GaN is deteriorated. Finally fully self-consistent GW gives the best agreement with experimental data.

In Fig. 1 the band structures for $\mathrm{AlN}, \mathrm{GaN}$, and $\mathrm{ZnO}$ within GGA, GGA $+U$ (for $\mathrm{GaN}$ and $\mathrm{ZnO}$ ) and $\mathrm{HSE}$ are shown. The shape of the bands is similar for all compounds but their positions are different. For GaN and $\mathrm{ZnO}$ both $\mathrm{HSE}$ and $\mathrm{GGA}+U$ gives similar position of cation semicore $d$ states - they are pushed down about $2 \mathrm{eV}$ - but the band gaps are closer to experimental in the hybrid functional scheme. This could be connected 

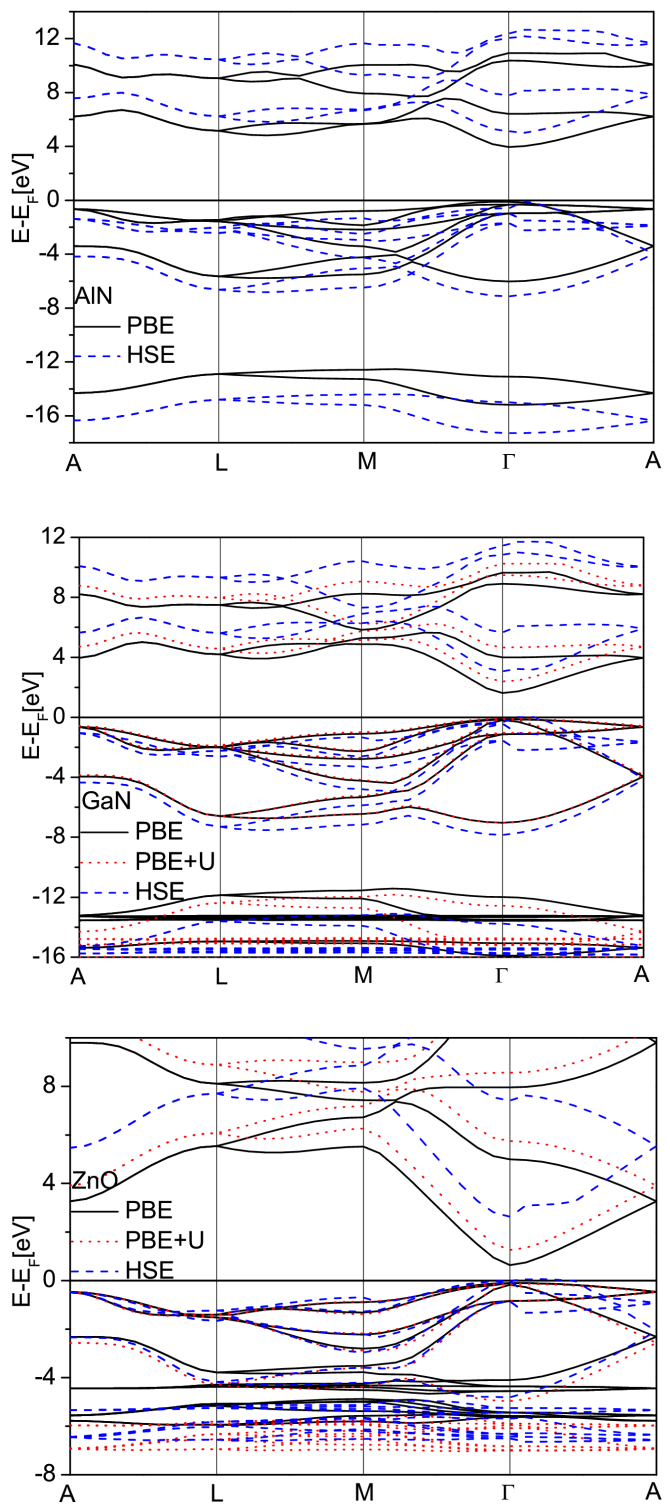

Fig. 1. Band structures of $\mathrm{AlN}, \mathrm{GaN}$ and $\mathrm{ZnO}$ within GGA (black, solid), GGA $+U$ (red, dotted) and HSE (blue, dashed) approximations.

TABLE III

Calculated direct band gaps for $\mathrm{ZnO}$, AlN and GaN within DFT and different GW approximations. Experimental values are taken from [11].

\begin{tabular}{c|c|c|c|c|c}
\hline \hline \multicolumn{6}{c}{ Band gap [eV] } \\
\hline & DFT & $\mathrm{G}_{0} \mathrm{~W}_{0}$ & $\mathrm{GW}_{0}$ & GW & Exp. \\
\hline $\mathrm{ZnO}$ & 0.793 & 2.334 & 2.871 & 3.640 & 3.430 \\
$\mathrm{AlN}$ & 4.095 & 5.714 & 5.780 & 6.226 & 6.190 \\
$\mathrm{GaN}$ & 1.774 & 3.348 & 3.110 & 3.448 & 3.500
\end{tabular}

with the fact that GGA $+U$ approach works mainly for $d$ electrons but HSE works for all states. To obtain similar results from $\mathrm{GGA}+U$ and HSE, the former should be applied also for $p$ electrons.

\section{Conclusions}

In the paper the electronic and crystallographic properties were calculated within different exchange-correlation approximations. The best agreement with experimental lattice constant was obtained by using hybrid functional. For the band gap the best results give full self-consistent GW approximation, however hybrid functional improves both band gap and positions of $d$ level on energy scale.

\section{Acknowledgments}

This work was supported by the Polish Ministry of Science and Higher Education as a research project No. N N202 288238.

\section{References}

[1] P. Hohenberg, W. Kohn, Phys. Rev. 136, B864 (1964).

[2] W. Kohn, L.J. Sham, Phys. Rev. 140, A1133 (1965).

[3] J.P. Perdew, A. Zunger, Phys. Rev. B 23, 5048 (1981).

[4] J.P. Perdew, K. Burke, M. Ernzerhof, Phys. Rev. Lett. 77, 3865 (1996).

[5] V.I. Anisimov, F. Aryasetiawan, A.I. Lichtenstein, J. Phys., Condens. Matter 9, 767 (1997).

[6] M. Shishkin, G. Kresse, Phys. Rev. B 74, 035101 (2006) and Phys. Rev. B 75, 235102 (2007) and references therein.

[7] J.P. Perdew, M. Ernzerhof, K. Burke, J. Chem. Phys. 105, 9982 (1996).

[8] J. Heyd, G.E. Scuseria, M. Ernzerhof, J. Chem. Phys. 118, 8207 (2003).

[9] G. Kresse, J. Furthmueller, Phys. Rev. B 54, 11169 (1996).

[10] G. Kresse, D. Joubert, Phys. Rev. B 59, 1758 (1999).

[11] A. Janotti, D. Segev, Ch.G. Van de Walle, Phys. Rev. B 74, 045202 (2006).

[12] P.G. Moses, M. Miao, Q. Yan, Ch.G. Van de Walle, J. Chem. Phys. 134, 084703 (2011).

[13] F. Oba, A. Togo, I. Tanaka, J. Paier, G. Kresse, Phys. Rev. B 77, 245202 (2008).

[14] A. Rubio, J.L. Corkill, M.L. Cohen, E.L. Shirley, S.G. Louie, Phys. Rev. B 48, 11810 (1993).

[15] M. Usuda, N. Hamada, T. Kotani, M. van Schilfgaarde, Phys. Rev. B 66, 125101 (2002). 